\title{
Effects of Diameters on Countercurrent Flow Limitation at a Square Top End in Vertical Pipes
}

\author{
Michio Murase $\mathbb{D}^{1},{ }^{1}$ Koji Nishida, ${ }^{1}$ Toshihide Torige, ${ }^{1}$ Toshiya Takaki, \\ Raito Goda, ${ }^{2}$ and Akio Tomiyama ${ }^{2}$ \\ ${ }^{1}$ Institute of Nuclear Technology, Institute of Nuclear Safety System, Inc., 64 Sata, Mihama-cho, Mikata-gun, Fukui 919-1205, Japan \\ ${ }^{2}$ Graduate School of Engineering, Kobe University, 1-1 Rokkodai, Nada-ku, Kobe-shi, Hyogo 657-8501, Japan \\ Correspondence should be addressed to Michio Murase; murase@inss.co.jp
}

Received 14 September 2018; Accepted 15 November 2018; Published 2 December 2018

Academic Editor: Tomoaki Kunugi

Copyright (C) 2018 Michio Murase et al. This is an open access article distributed under the Creative Commons Attribution License, which permits unrestricted use, distribution, and reproduction in any medium, provided the original work is properly cited.

\begin{abstract}
The falling liquid flow rate under flooding conditions is limited at a square top end of a vertical pipe in the pressurizer surge line with the diameter of about $300 \mathrm{~mm}$ that consists of a vertical pipe, a vertical elbow, and a slightly inclined pipe with elbows. In this study, therefore, we evaluated effects of diameters on countercurrent flow limitation (CCFL) at the square top end in vertical pipes by using existing air-water data in the diameter range of $D=19-250 \mathrm{~mm}$. As a result, we found that there was a strong relationship between the constant $C_{\mathrm{K}}$ and the slope $m$ in the Wallis-type correlation where the Kutateladze parameters were used for the dimensionless gas and liquid velocities. The constant $C_{\mathrm{K}}$ and the slope $m$ increased when the water level is increased in the upper tank $h$. CCFL at the square top end of the vertical pipes could be expressed by the Kutateladze parameters with $C_{\mathrm{K}}=1.53 \pm 0.11$ and $m=0.97$ for $D \geq 30 \mathrm{~mm}$. The $C_{\mathrm{K}}$ values were smaller for $D=19-25 \mathrm{~mm}$ than those for $D \geq 30 \mathrm{~mm}$.
\end{abstract}

\section{Introduction}

Under postulated accident conditions such as loss-of-coolant accidents (LOCAs) in pressurized water reactors (PWRs), steam and condensate water form countercurrent flows in a hot leg (consisting of a horizontal pipe, a 50-deg vertical elbow, and a short inclined pipe) and a pressurizer surge line (consisting of a slightly inclined pipe with elbows, a vertical elbow, and a vertical pipe), and flooding may occur. For transient and accident analyses of PWRs, characteristics of countercurrent flow limitation (CCFL) should be considered strictly, where CCFL is defined by the relationship between the time-averaged gas superficial velocity and falling liquid superficial velocity, $J_{\mathrm{G}}$ and $J_{\mathrm{L}}$, under flooding conditions, because the falling liquid flow rate affects the water mass in the reactor core and cooling of the fuel rods.

Previously, we (Murase et al. [1]) developed a onedimensional computation method with parameters adjusted from CCFL data in hot leg and pressurizer surge line models (Mayinger et al. [2]; Minami et al. [3]; Futatsugi et al. [4]), and we could predict CCFL in nearly horizontal pipes for the hot leg and pressurizer surge line within a practical uncertainty. There are only a few CCFL experiments simulating the pressurizer surge line with a diameter of about $300 \mathrm{~mm}$. In available data (Takeuchi et al. [5]; Futatsugi et al. [4]; Yu et al. $[6,7])$, the pipe diameters were $D=30-90 \mathrm{~mm}$, so that the CCFL data were not sufficient to evaluate effects of the diameters. In the pressurizer surge line, the falling liquid flow rate is limited at the square top end of the vertical pipe, and so we (Yamamoto et al. [8]) derived a correlation for CCFL-U at the square top end for the pressurizer surge line by using existing data for $D=19-140 \mathrm{~mm}$ (Richter [9]; Doi et al. [10]). However, CCFL-U behavior is very complex and there are some disagreements among experimental data. Therefore, it is important to evaluate effects of the diameters on CCFL-U at the square top end of vertical pipes and to apply these results to the pressurizer surge line.

In this study, we evaluated effects of diameters on CCFL$\mathrm{U}$ at the square top end in vertical pipes by using existing air-water data reported by Richter [9] for $D=19-140 \mathrm{~mm}$, Doi et al. [10] for $D=30-60 \mathrm{~mm}$, Wallis and Kuo [11] for $D$ $=19-145 \mathrm{~mm}$, Bharathan et al. [12] for $D=19-250 \mathrm{~mm}$, and 


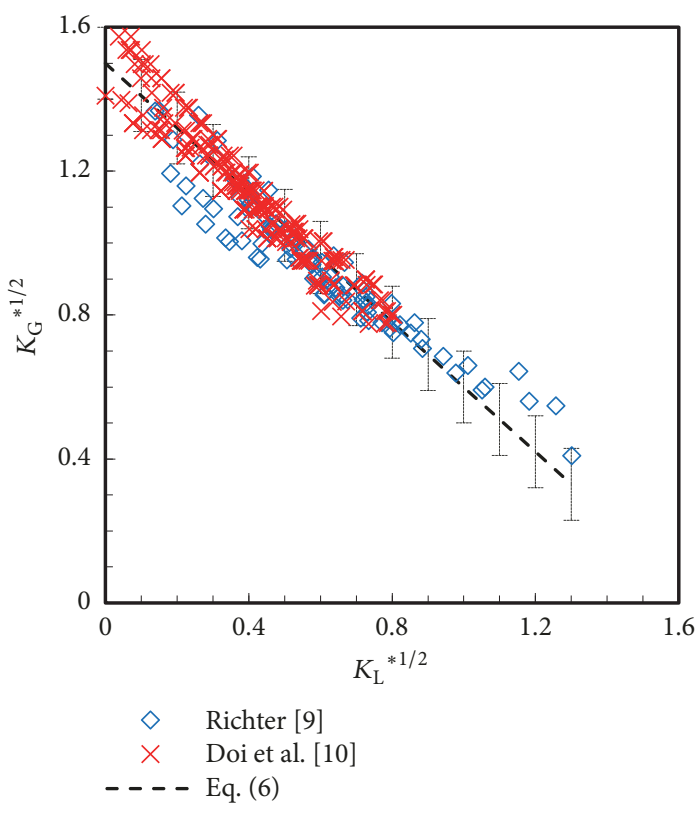

(a)

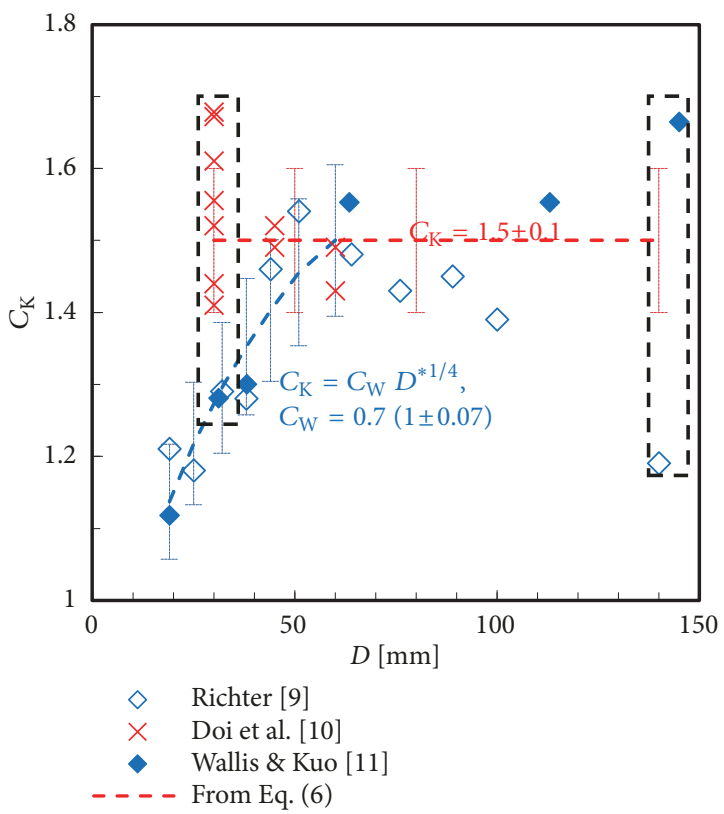

(b)

FIGURE 1: CCFL characteristics at square top ends: (a) CCFL expressed by Kutateladze parameters and (b) CCFL constant (broken-line box: disagreement between data).

Matsumura and Kaminaga [13] for $D=20 \mathrm{~mm}$. From data by Richter [9], Wallis and Kuo [11], and Bharathan et al. [12], CCFL-U for large diameters was evaluated. The effects of the water level in the upper tank were evaluated from data mainly by Doi et al. [10], and the difference between data by Richter [9] and Doi et al. [10] was discussed. From the results, CCFL$\mathrm{U}$ characteristics were classified depending on the diameter, the shape of the top end, and the water level in the upper tank, and features of CCFL-U were summarized. All data used in this study were obtained under air-water conditions at atmospheric pressure.

\section{Previous Studies on CCFL at the Square Top End}

2.1. General Form of CCFL Correlation. For safety analyses during transients and accidents in nuclear power plants, the CCFL correlation by Wallis [15] has often been applied to evaluate the falling liquid flow rate. The general form of the Wallis correlation is given by

$$
\begin{aligned}
H_{\mathrm{G}}^{* 1 / 2}+m H_{\mathrm{L}}^{* 1 / 2} & =C_{i}, \quad(i=\mathrm{K} \text { or } \mathrm{W}) \\
H_{k}^{*} & =J_{k}\left\{\frac{\rho_{k}}{g w\left(\rho_{\mathrm{L}}-\rho_{\mathrm{G}}\right)}\right\}^{1 / 2}, \\
& (k=\mathrm{G} \text { or } \mathrm{L})
\end{aligned}
$$

where $g\left[\mathrm{~m} / \mathrm{s}^{2}\right]$ is the gravitational acceleration, $J[\mathrm{~m} / \mathrm{s}]$ is the superficial velocity, $H^{*}[-]$ is the dimensionless velocity, $w[\mathrm{~m}]$ is the characteristic length, and $\rho\left[\mathrm{kg} / \mathrm{m}^{3}\right]$ is the density. The slope $m$ and the constant $C_{i}$ are determined from experiments, where the subscripts $\mathrm{K}$ and $\mathrm{W}$ show the Kutateladze parameter and Wallis parameter for $\mathrm{H}_{k}{ }^{*}$, respectively. Bankoff et al. [16] defined the characteristic length $w$ by

$$
\begin{aligned}
& w=D^{(1-\beta)} L^{\beta}, \quad 0 \leq \beta \leq 1, \\
& L=\left[\frac{\sigma}{\left\{g\left(\rho_{\mathrm{L}}-\rho_{\mathrm{G}}\right)\right\}}\right]^{1 / 2}
\end{aligned}
$$

where $D[\mathrm{~m}]$ is the diameter, $L[\mathrm{~m}]$ is the Laplace capillary length, and $\sigma[\mathrm{N} / \mathrm{m}]$ is the surface tension. In $(1), H^{*}$ indicates the Wallis parameter $J^{*}$ at $\beta=0$, while $H^{*}$ is the Kutateladze parameter $K^{*}$ at $\beta=1 . J^{*}$ and $K^{*}$ can be converted to each other by using $D^{*}$, as follows:

$$
J^{*}=\frac{K^{*}}{D^{* 1 / 2}}, \quad D^{*}=\frac{D}{L} .
$$

Equations (2) and (3) show primary parameters for the length scale $(D$ or $L)$ and fluid properties ( $\rho$ and $\sigma$ ). However, it is well known that $w$ in (3) changes depending on $D$ in vertical pipes (Wallis and Makkenchery [17]) and that the liquid viscosity (which is not included in (2) and (4)) affects CCFL in vertical pipes (Wallis [15]).

2.2. CCFL Characteristics and Technical Issues. Figure 1 shows CCFL characteristics at the square top end in vertical pipes. CCFL data reported by Richter [9] and Doi et al. [10] are well expressed by the Kutateladze parameters. From the data of $D$ $=19-140 \mathrm{~mm}$ in Figure 1(a), the Wallis-type correlation (6) was derived using the least-square method (Yamamoto et al. [8]).

$$
K_{\mathrm{G}}^{* 1 / 2}+0.9 K_{\mathrm{L}}^{* 1 / 2}=1.5 \pm 0.1
$$

Figure 1(b) shows the CCFL constant $C_{\mathrm{K}}$, which was obtained for each experimental case using the least-square method. 
TABLE 1: Classifications of CCFL at the square top end (Region "R-": cf. Figure 8(a)).

\begin{tabular}{lcccc}
\hline Classification & Top end & $D[\mathrm{~mm}]$ & $h$ [mm] & Data base \\
\hline Basic, pe (R-I) & Protruding & $44,51,64,76,89,100$ & Low & Richter [9] \\
\hline Basic, se (R-I) & Square & $30,45,60$ & 100,200 & Doi et al. [10] \\
& & 51 & Mid-level & Bharathan et al. [12] \\
\hline High $h$, se (R-II) & Square & 30 & $300,450,600$ & Doi et al. [10] \\
\hline Large $D$, se (R-II) & Square & 148 & Mid-level & Bharathan et al. [12] \\
\hline Large $D$, pe (R-III) & Protruding & 140 & Low & Richter [9] \\
& & 148 & Mid-level & Bharathan et al. [12] \\
& & 250 & Mid-level & Richter et al. [14] \\
\hline Low $h$, pe (R-III) & Protruding & $19,25,32,38$ & Low & Richter [9] \\
\hline Small $D$, pe (R-IV) & Protruding & 19 & Mid-level & Bharathan et al. [12] \\
\hline Small $D$, se (R-IV) & Square & 19,25 & Mid-level & Bharathan et al. [12] \\
& & 20 & 50, 100, 200 & Matumura and Kaminaga [13] \\
\hline
\end{tabular}

Data by Wallis and Kuo [11] are not the CCFL constant but the zero water penetration (ZWP), which generally agrees with $C_{\mathrm{K}}$. Technical issues are disagreements between data by Richter [9] and Doi et al. [10] for $D=$ about $30 \mathrm{~mm}$, and data by Richter [9] and Wallis and Kuo [11] for $D=$ about 140 $\mathrm{mm}$. The $C_{\mathrm{K}}$ values by Richter [9] and the ZWP values by Wallis and Kuo [11] suggest that CCFL characteristics might be expressed by the Wallis parameter $\left(C_{\mathrm{W}}=0.7\right)$ for the region of small diameters of $D<40 \mathrm{~mm}$, but this should be confirmed from CCFL data.

2.3. Experimental Conditions in Previous Studies. Figure 2 shows the shapes near the top ends of the vertical pipes used in previous experiments, and Table 2 lists major experimental conditions. Wallis and Kuo [11] used a protruding top end (Figure 2(a)), and the water level in the upper tank was low ( $h<7 \mathrm{~mm}$ ), where the gas core continued from the lower tank to the atmosphere. Bharathan et al. [12] used a square top end (Figure 2(b)) and also a protruding top end, and the water level in the upper tank was midlevel, where the upper part of the top end was covered by the air-water mixture. Richter [9] referred to CCFL data by Wallis and Makkenchery [17], but we could not find the CCFL data in Wallis and Makkenchery [17]. Therefore, we do not know the shape near the top end of the vertical pipe reported by Richter [9]. From the comparison of data by Richter [9] with data by Wallis and Kuo [11] and Bharathan et al. [12], we assumed that a protruding top end was used and the water level was low. Matsumura and Kaminaga [13] used a square top end with a horizontal plate (Figure 2(c)) to ensure clear observations. Doi et al. [10] used a square top end with three upper tanks (Figure 2(d)): a rectangular tank (UT1), a circular tank (UT2), and a cylindrical tank (UT3). All experiments [9-13] listed in Table 2 were done under air-water conditions at the room temperature and atmospheric pressure.

\section{CCFL Characteristics at the Square Top End}

3.1. CCFL in Vertical Pipes of Large Diameters. Our main interest is CCFL in large diameters for application to pressurizer surge lines with $D=$ about $300 \mathrm{~mm}$. Bharathan et al.
[12] reported some air-water data with such large diameters. Figure 3 compares CCFL data in large diameters with (6). The single data point by Wallis and Kuo [11] is for the zero water penetration with the protruding top end. The data by Richter [9] and Wallis and Kuo [11] agreed well with data by Bharathan et al. [12] with the protruding top end. Hence the data by Richter [9] might be measured with the protruding top end. This was the reason why we judged the top end in the experiment by Richter [9] to be "protruding" in Table 2. The difference between data points by Richter [9] and Wallis and Kuo [11] for $D=140-145 \mathrm{~mm}$ in Figure 1(b) was due to the small slope in the middle range of $K_{\mathrm{G}}{ }^{* 1 / 2}$ and the large slope in the large range of $K_{\mathrm{G}}{ }^{* 1 / 2}$.

Our interest is not CCFL with the protruding top end but CCFL with the square top end. $K_{\mathrm{L}}{ }^{* 1 / 2}$ values with the square top end are larger in the wide range of $K_{\mathrm{G}}{ }^{* 1 / 2}$ than those with the protruding top end. Figure 3 shows that (6) can be applied to large diameters up to $D=250 \mathrm{~mm}$ (Richter et al. [14]).

3.2. Effects of Water Level in Upper Tank on CCFL. We (Doi et al. [10]) measured CCFL characteristics at the square top end using the three upper tanks shown in Figure 2(d). Figure 4 shows the effects of the upper tanks and water levels there on the CCFL constant $C_{\mathrm{K}}$ and the slope $m$. As shown in Figure 4(a), the shape of the upper tanks affected $C_{K}$ and $m$ due to different sloshing behavior there and pressure fluctuation in the lower tank, which affected fluctuation of the air flow rate into the vertical pipe from the lower tank. Clear effects of the diameters on $C_{\mathrm{K}}$ and $m$ were not observed, and effects of the upper tanks on $C_{\mathrm{K}}$ and $m$ were not significant.

As shown in Figure 4(b), $C_{\mathrm{K}}$ and $m$ with $D=30 \mathrm{~mm}$ increased with increasing the water level $h$ in the upper tank (Doi et al. [10]). As decreasing $h, C_{\mathrm{K}}$ and $m$ by Doi et al. [10] approached those with $D=32 \mathrm{~mm}$ by Wallis and Kuo [11] with a low water level and Richter [9]. This was the reason we judged that the water level in the experiment by Richter [9] was low in Table 2. Matsumura and Kaminaga [13] reported that clear differences were not measured for $D=20 \mathrm{~mm}$ and $h=0.05-0.2 \mathrm{~m}$. Therefore, the effects of the water levels may depend on the shape of the upper tank and pipe diameter. 


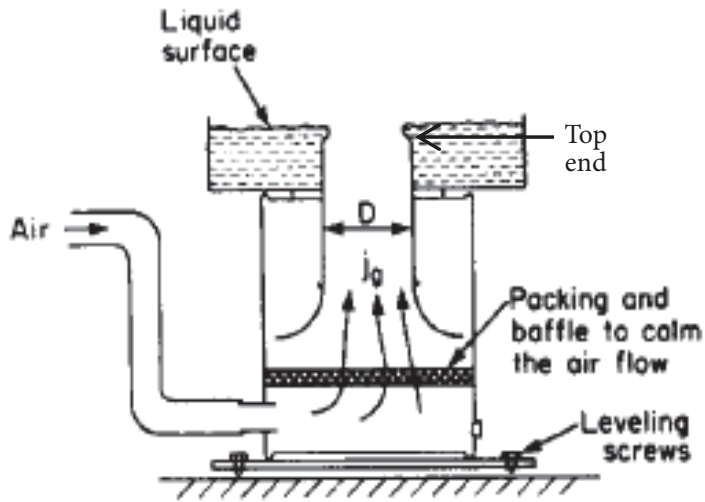

(a)

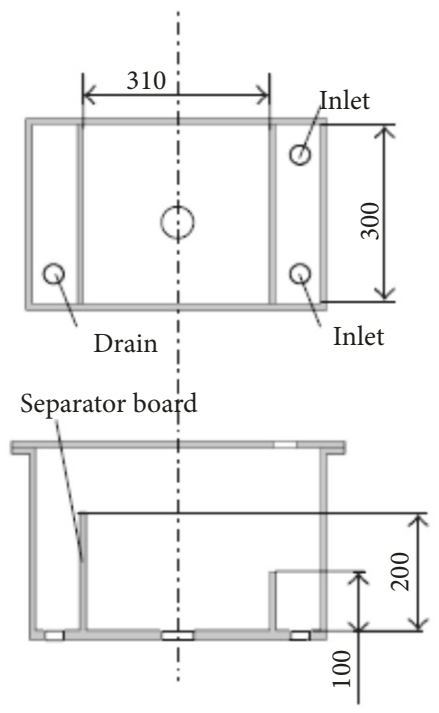

Rectangular tank (UT1)

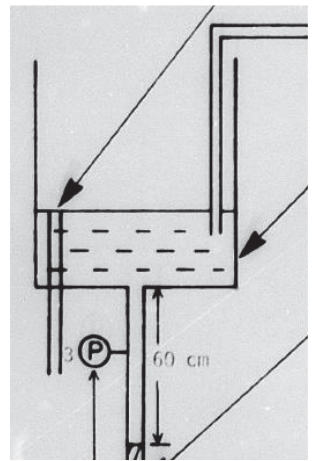

(b)

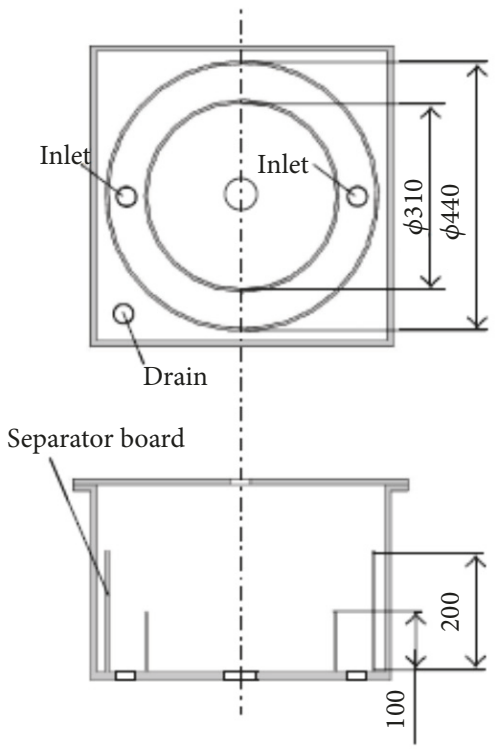

Circular tank (UT2)

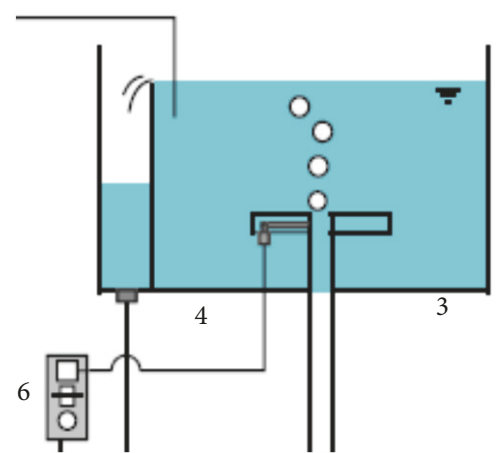

(c)

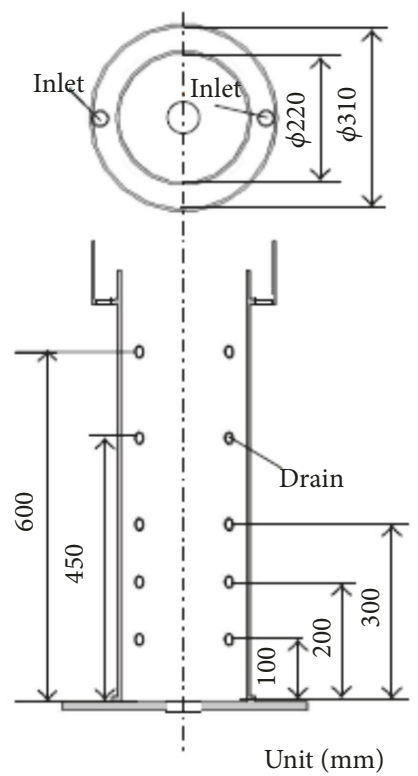

Cylindrical tank (UT3)

(d)

Figure 2: Shapes near the top end of the vertical pipe used by (a) Wallis and Kuo [11], (b) Bharathan et al. [12], (c) Matsumura and Kaminaga [13], and (d) Doi et al. [10].

Figure 4 suggests a clear interrelation between $C_{\mathrm{K}}$ and $m$, which will be discussed later.

3.3. CCFL in Vertical Pipes of Small Diameters. As shown in Figure 1(b), there was clear disagreement between the data by Richter [9] and Doi et al. [10] for $D=$ about $30 \mathrm{~mm}$, and $C_{\mathrm{K}}$ values by Richter [9] and ZWP by Wallis and Kuo [11] suggested that CCFL characteristics might be expressed by the Wallis parameter $\left(C_{\mathrm{W}}=0.7\right)$ in the region of $D<40 \mathrm{~mm}$. The large difference of $C_{\mathrm{K}}$ values by Doi et al. [10] for $D=$ about $30 \mathrm{~mm}$ in Figure 1(b) was due to effects of the water level in the upper tank as shown in Figure 4(b).

Figure 5 shows CCFL characteristics for (a) medium and (b) small diameters. For the medium diameters of $D=44$ $51 \mathrm{~mm}$, all data points were within the uncertainty of (6) as shown in Figure 5(a). For $D=51 \mathrm{~mm}$, the protruding or square end and the water level at low or midlevel (cf. Table 2) did not affect CCFL. For the small diameters of $D=19-25$ $\mathrm{mm}$, CCFL characteristics were clearly different from (6) as shown in Figure 5(b). The protruding or square end did not affect CCFL from the $19 \mathrm{~mm}$ diameter data by Bharathan et al. [12]. The slope of data with the low water level by Richter [9] $(D=19 \mathrm{~mm})$ was clearly smaller than that of other data with the water level at midlevel. This suggests that the small $C_{\mathrm{K}}$ values for the small diameters of $D=19-38 \mathrm{~mm}$ by Richter [9] and Wallis and Kuo [11] in Figure 1(b) might be due to the low water level. For data with the square top end, the slope was similar to that of (6) and there were no clear trends for diameters.

Figure 6 shows CCFL characteristics in small diameters with low water levels by Richter [9]. As shown in Figure 6(a), the slope $m$ could be expressed by an exponent function of the dimensionless diameter $D^{*}$ as $m=0.16 D^{* 0.58}$. Figure 6(b) shows CCFL data plotted for $J_{\mathrm{G}}{ }^{* 1 / 2}$ and $D^{* 0.58} J_{\mathrm{L}}{ }^{* 1 / 2}$. From 
TABle 2: Experimental conditions ( $D$, diameter; $h$, water level; $*$ our judgement of the condition).

\begin{tabular}{lccc}
\hline Reference & Top end & $D[\mathrm{~mm}]$ & $h[\mathrm{~mm}]$ \\
\hline Wallis and Kuo [11] & Protruding & $6.4,19,31.2,38.2$, & $<7$ \\
& & $63.5,113,145$ & Mid-level \\
Bharathan et al. [12] & Protruding & $19,148,250$ & $(<7) *$ \\
& Square & $19,25,51,148$ & \\
Richter [9] & (Protruding)* & $19,25,32,38,44,51$, & $50,100,200$ \\
& & $64,76,89,100,140$ & 12,20 \\
Matsumura and & Square & $30,45,60$ (UT1) & 200 \\
Kaminaga [13] & & $30,45,60$ (UT2) & 200 \\
Doi et al. [10] & Square & 30 (UT3) & $100,200,300,450,600$ \\
& &
\end{tabular}

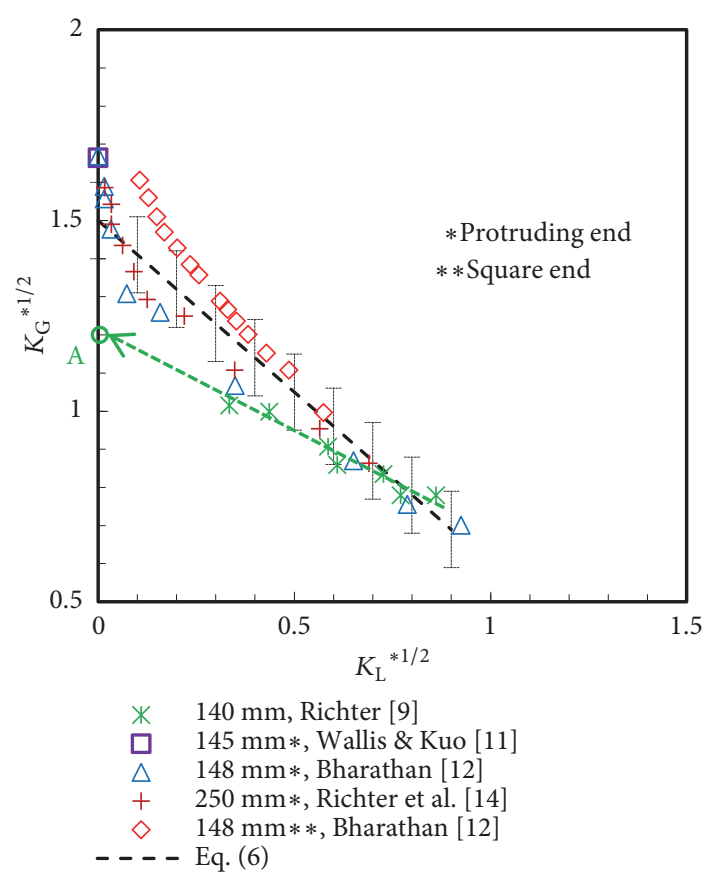

Figure 3: CCFL characteristics for large diameters and effects of protruding end (A: small $C_{\mathrm{K}}$ value by Richter [9] in Figure 1(b)).

the data points in Figure 6(b), the CCFL correlation was derived using the least-square method as

$$
K_{\mathrm{G}}^{* 1 / 2}+0.16 D^{* 0.58} K_{\mathrm{L}}^{* 1 / 2}=0.71 \pm 0.05
$$

All data points in the diameter range of $D=19-44 \mathrm{~mm}$ were within the small uncertainty of \pm 0.05 . The data points for the diameter of $D=44 \mathrm{~mm}$ were also within the uncertainty of (6) as shown in Figure 5(a).

3.4. Features of CCFL at the Square Top End. Figure 7 shows the constant $C_{K}$ and the slope $m$ in (1) for CCFL at the square top end. The $C_{\mathrm{K}}$ and $m$ values differed largely depending on the shape of the top end (square or protruding), the diameter, and the water level in the upper tank. The difference was mainly due to the shape of the top end (square or protruding) in the large diameters of $D^{*}>37(D>100 \mathrm{~mm})$ and the water level in the upper tank in the small diameters of $D^{*}<16(D$ $<44 \mathrm{~mm}$ ). This means that the shape of the top end and water level did not significantly affect CCFL in the diameter range of $D=44-100 \mathrm{~mm}\left(D^{*}=16-37\right)$.

CCFL characteristics depended on the shape of the top end (square or protruding), diameters, and the water level in the upper tank, and Table 1 lists classifications of CCFL. Figure 8 shows features of CCFL characteristics at the square top end (including the protruding top end). As shown in Figure 8(a), a clear relationship between $C_{\mathrm{K}}$ and $m$ was found except for the region "R-IV" (small diameters of $D=19$ $25 \mathrm{~mm}$ with the water level at midlevel). The relationship between $C_{\mathrm{K}}$ and $m$ was expressed by

$$
\begin{aligned}
C_{\mathrm{K}}=0.52 \ln (m)+ & 1.54 \pm 0.05 \\
& \left(1.19 \leq C_{\mathrm{K}} \leq 1.7,0.5 \leq m \leq 1.4\right) .
\end{aligned}
$$

In region "R-I", the CCFL characteristics $\left(C_{\mathrm{K}}=1.5 \pm 0.1\right.$ and $m=0.88 \pm 0.13$ ) were measured with $D=30-100 \mathrm{~mm}$ and $h \leq$ $200 \mathrm{~mm}$ or midlevel (cf. "Basic" in Table 1). On increasing the diameter or the water level with the square top end, $C_{\mathrm{K}}$ and $m$ increased (region "R-II"). In region "R-III", all data were obtained with the protruding top end. $C_{\mathrm{K}}$ and $m$ in region "RIV" with the small diameters of $D=19-25 \mathrm{~mm}$ and the water level at midlevel were outside the range of values calculated by using (8). Our major interest is CCFL in large diameters with the square top end, and therefore all data in Regions " $\mathrm{R}$ I" and "R-II" and data with large diameters in region "R-III" are plotted in Figure 8(b). From data points in Figure 8(b), we derived the CCFL correlation using the least-square method as follows:

$$
\begin{aligned}
K_{\mathrm{G}}^{* 1 / 2}+0.97 K_{\mathrm{L}}^{* 1 / 2}=1.53 \pm 0.11 \\
\left(30 \mathrm{~mm} \leq D \leq 250 \mathrm{~mm}, 11 \leq D^{*} \leq 94\right)
\end{aligned}
$$

$95 \%$ of the 290 data points were within the uncertainty of \pm 0.11 . Some data points at large $J_{\mathrm{G}}{ }^{* 1 / 2}$ with $D=148 \mathrm{~mm}$ and the square top end and some data points at medium $J_{\mathrm{G}}{ }^{* 1 / 2}$ with the protruding top end, which were larger and smaller than (9), respectively, were outside the uncertainty of \pm 0.11 . 


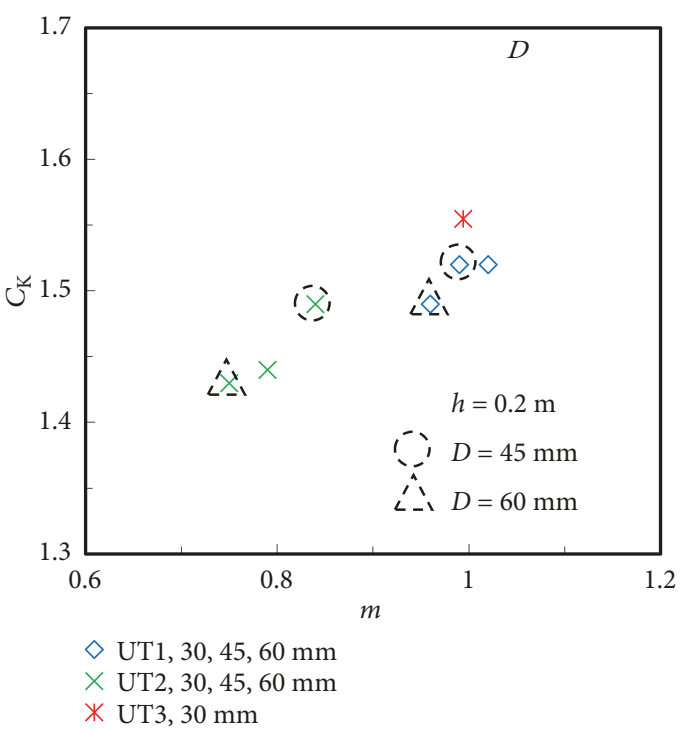

(a)

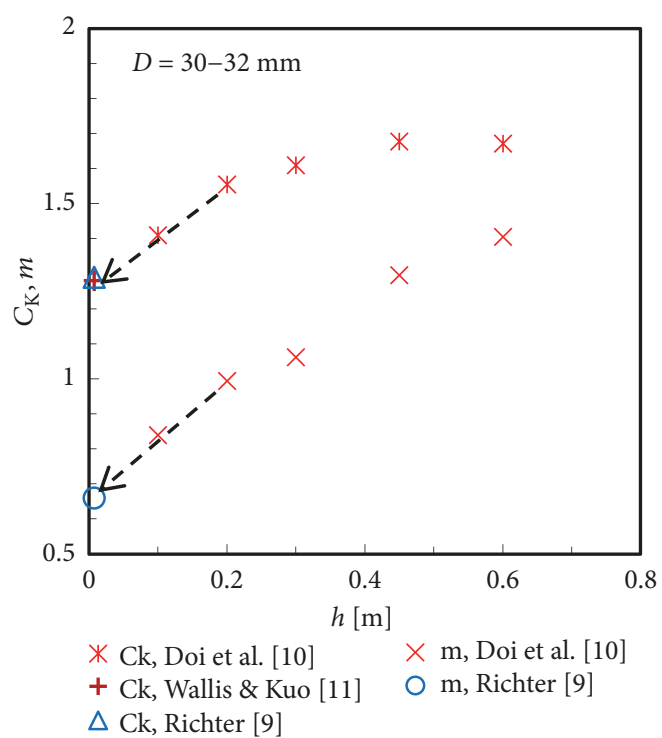

(b)

Figure 4: Effects of (a) upper tank by Doi et al. [10] (UT1-UT3: cf. Figure 2(d)) and (b) water level $h$ on $C_{\mathrm{K}}$ and $m$.

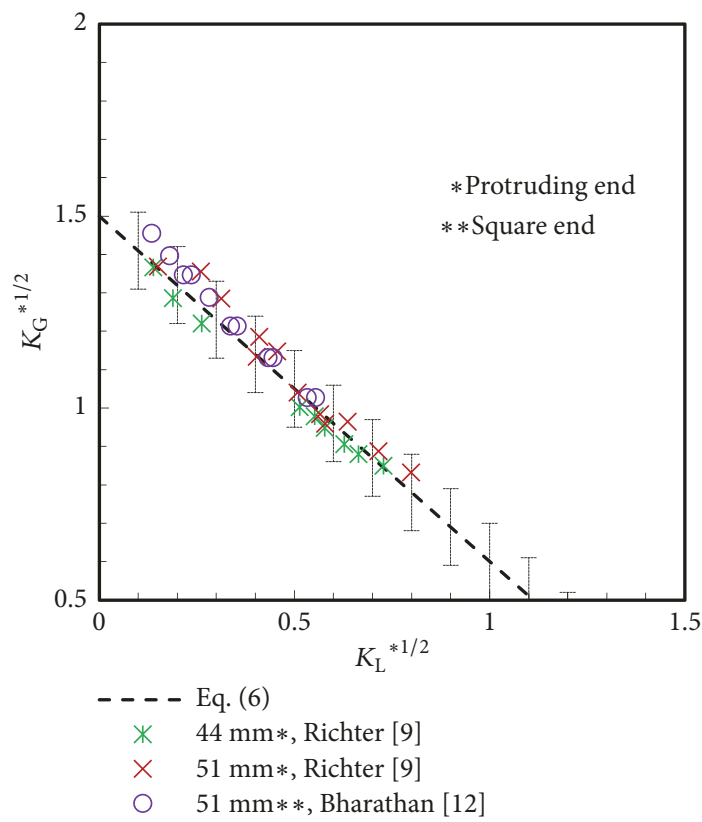

(a)

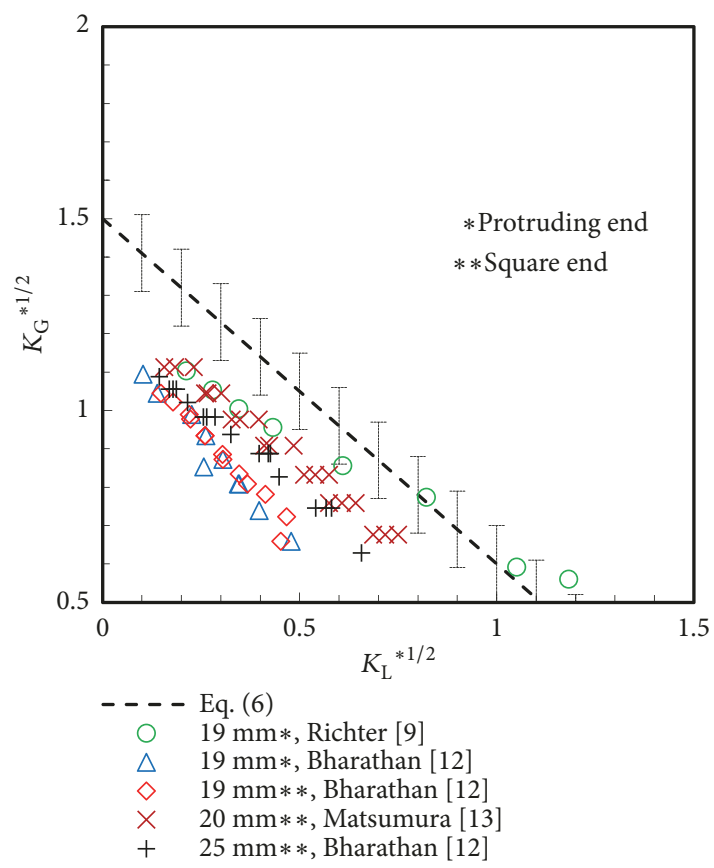

(b)

FiguRE 5: CCFL characteristics in (a) medium diameters and (b) small diameters with water levels at midlevel (except $D=19 \mathrm{~mm}$ by Richter [9]).

Though the difference between (6) and (9) was small, we recommend using (9) because of the wide range of CCFL data used for its derivation.

Table 3 summarizes effects of parameters on CCFL at the square top end. Effects of "Large", "Medium", and "Small" were determined by comparison with conditions of the square top end and water level at midlevel for $D=30-100 \mathrm{~mm}$ where the uncertainty was $\pm 0.1(6.7 \%)$ for $C_{\mathrm{K}}=1.5$. Our target to improve a CCFL correlation was uncertainty smaller than $\pm 10 \%$ for the CCFL constant $C_{\mathrm{K}}$, and "Medium" was within the target of $\pm 10 \%$. "Large" was uncertainty larger than $\pm 10 \%$ and we recommend using a different CCFL correlation for "Large". However, the data with the protruding end for $D=$ 140-250 mm were included to derive (9) due to limited data for large diameters.

For low water levels, we recommend using the minimum of (7) for small diameters and (9) for medium to large diameters. For the small diameters of $19-25 \mathrm{~mm}$ with a 


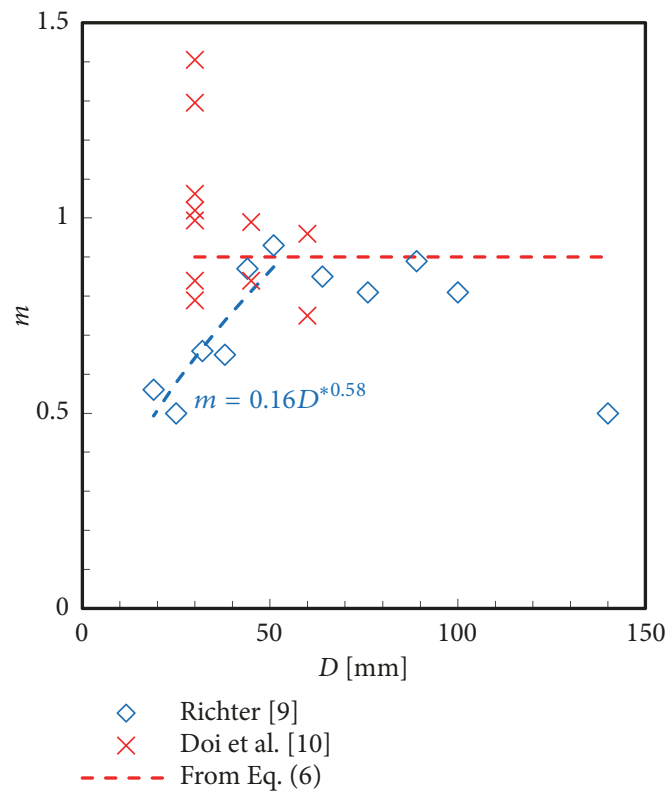

(a)

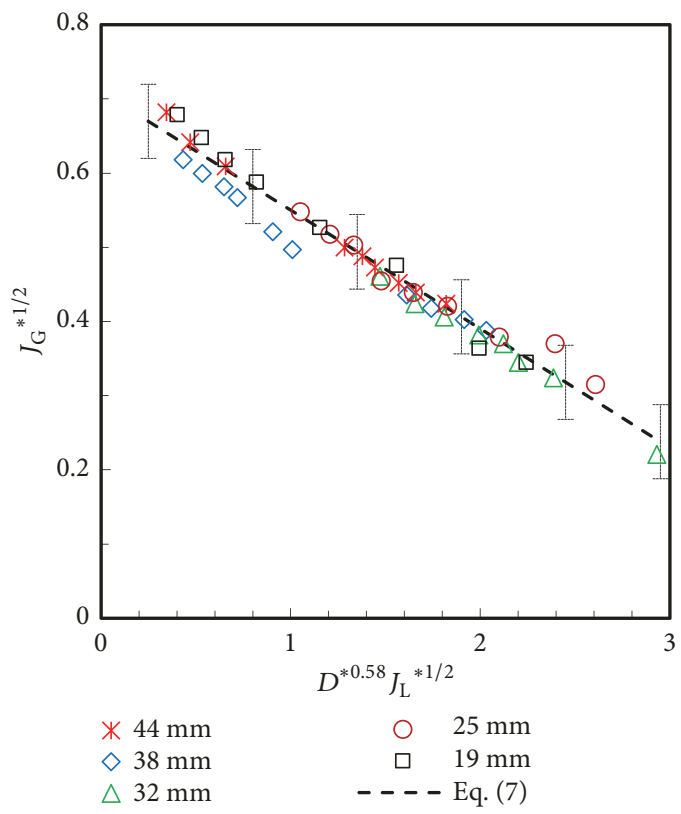

(b)

FIgURE 6: CCFL characteristics for small diameters with low water levels by Richter [9]: (a) slope $m$, and (b) CCFL.

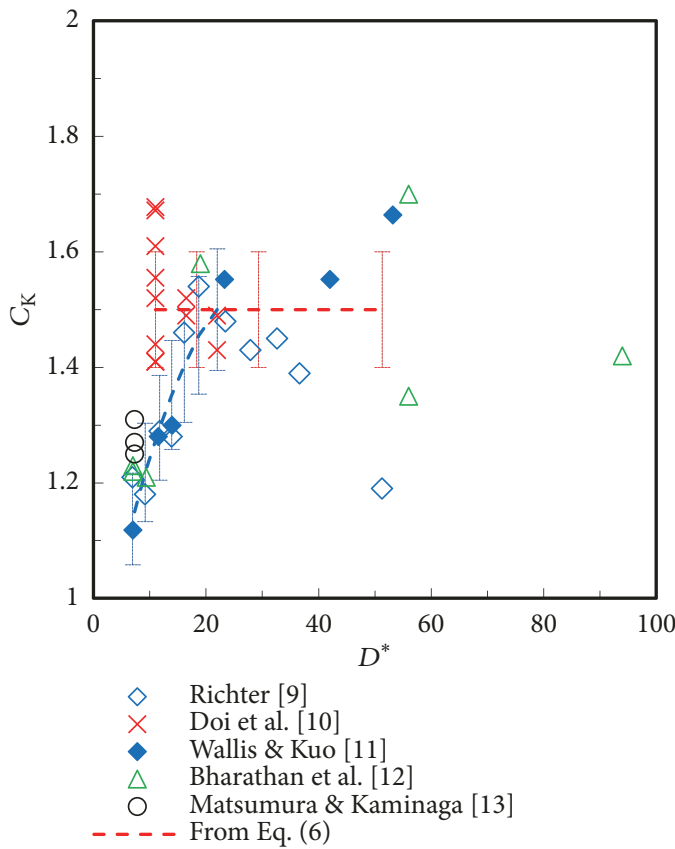

(a)

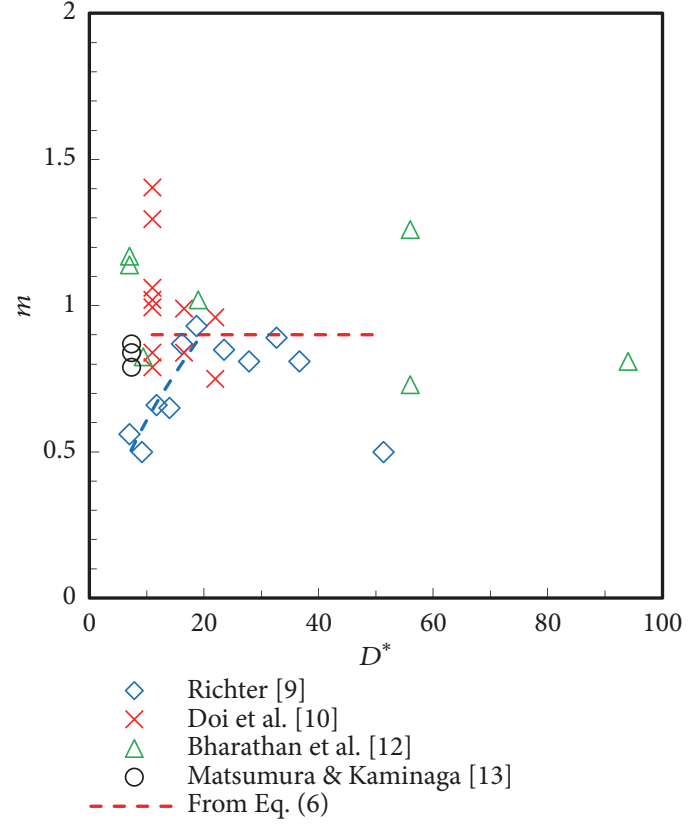

(b)

FIGURE 7: CCFL characteristics at the square top end: (a) CCFL constant $C_{\mathrm{K}}$ and (b) slope $m$.

square end, we recommend using a correlation specific for a diameter, because CCFL characteristics were different among the databases as shown in Figure 5(b).

\section{Discussion}

Our final target is to evaluate CCFL characteristics in an actual pressurizer surge line. Hence we have to evaluate effects of pressure and temperature on CCFL and also system characteristics of the pressurizer surge line. However, there are only a few CCFL experiments simulating the pressurizer surge line with the diameter of about $300 \mathrm{~mm}$. In available data (Takeuchi et al. [5]; Futatsugi et al. [4]; Yu et al. [6, 7]), the pipe diameters were $D=30-90 \mathrm{~mm}$, and CCFL data were not sufficient to evaluate effects of the diameters and fluid 
TABLE 3: Effects of parameters on CCFL at square top end comparing with conditions of the square top end and water level at midlevel for $D$ = 30-100 mm ("Medium" is uncertainty range of about 5-10 \% for $C_{\mathrm{K}}$, for "Large" different correlation is recommended, bold area; database used for (9)).

\begin{tabular}{llccc}
\hline$D[\mathrm{~mm}]$ & $19-25$ & $30-38$ & $44-100$ & $140-250$ \\
\hline $\begin{array}{l}\text { Diameter } \\
\text { (Square end) }\end{array}$ & Large & & Small (base case) & Medium \\
\hline Protruding end & & Small & Medium & Large \\
\hline Low water level & & Large & Small \\
\hline
\end{tabular}

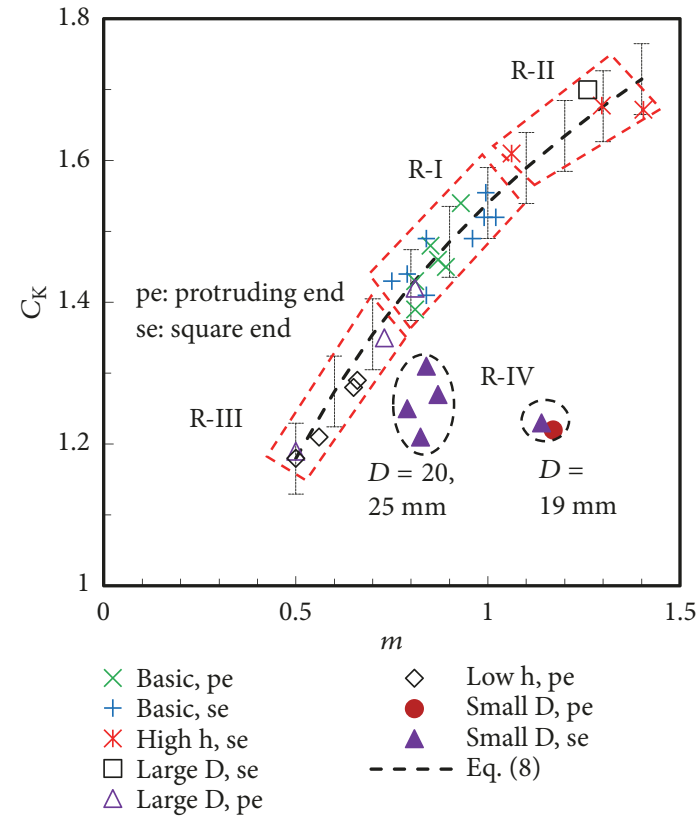

(a)

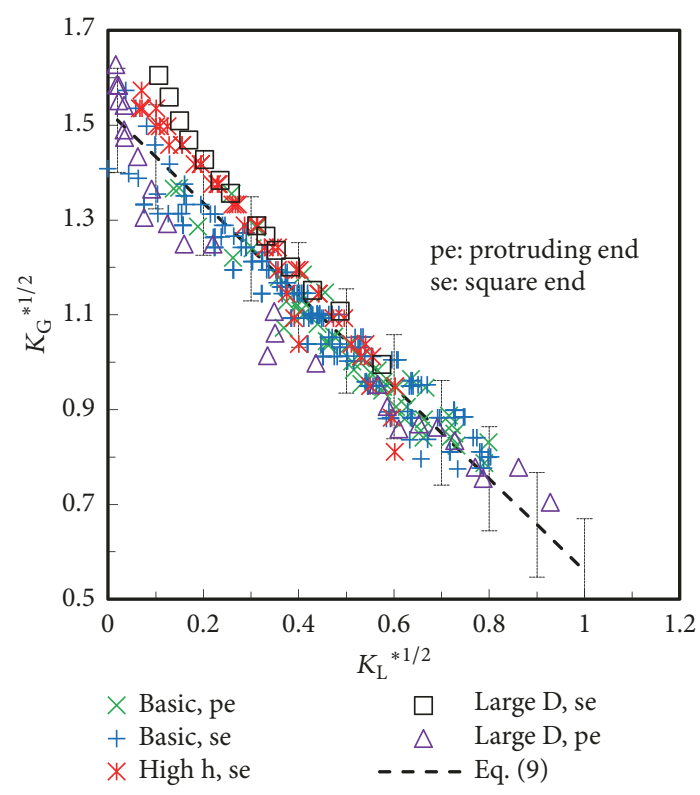

(b)

Figure 8: Features of CCFL at the square top end: (a) relationship between $C_{\mathrm{K}}$ and $m$ (classifications: cf. Table 1) and (b) CCFL for medium and large diameters.

properties. Therefore, the features of CCFL at the square top end discussed in Section 3.4 are important when we evaluate CCFL characteristics in the pressurizer surge line.

4.1. Effects of Pressure and Temperature. Ilyukhin et al. [18, 19] reported CCFL data with $D=20 \mathrm{~mm}$ at pressures of $P=0.6$ 4.1 $\mathrm{MPa}$ and with $D=30$ and $40 \mathrm{~mm}$ at $P=0.3-1.6 \mathrm{MPa}$, respectively. The data points for $D=30$ and $40 \mathrm{~mm}$ at $P=0.3$ $-1.6 \mathrm{MPa}$ obtained by Ilyukhin et al. [19] were plotted on the diagram of $K_{k}^{* 1 / 2} D^{* 1 / 8}\left(\rho_{G} / \rho_{L}\right)^{0.05}$. Since the data points for each pressure were not separated, we cannot evaluate $K_{k}{ }^{* 1 / 2}$ values from the data. They measured CCFL data with the protruding top and bottom ends at a high water level in the upper tank. Figure 8(a) and Table 3, therefore, may be useful for evaluating effects of fluid properties from the data by Ilyukhin et al. [19] with $D=20 \mathrm{~mm}$ at $P=0.6-4.1 \mathrm{MPa}$.

4.2. Computations with the Annular Flow Model. CCFL characteristics in vertical pipes can be computed by using the annular flow model with the maximum flow condition of $\left(\partial J_{\mathrm{L}}^{*} / \partial \alpha\right)=0$ or $\left(\partial J_{\mathrm{G}}^{*} / \partial \alpha\right)=0$ and correlations for the interfacial friction factor and the wall friction factor (Richter [9]; Bharathan and Wallis [20]; Sudo [21]; Yamamoto et al. [8]). In the computations, the correlation for the interfacial friction factor is important. Sudo [21] improved the correlation for the interfacial friction factor by Bharathan and Wallis [20] to obtain good agreement between computed results and data by Richter [9]. As shown in Figure 7(a), however, the $C_{K}$ value by Richter [9] had a maximum at an intermediate diameter and decreased with increasing the diameter toward large diameters due to the protruding end. The correlation for the interfacial friction factor improved by Sudo [21] gave a small $C_{\mathrm{K}}$ value for a large diameter (Yamamoto et al. [8]). Therefore, the correlation for the interfacial friction factor should be improved to apply it to a large diameter without a protruding end.

4.3. System Characteristics. Figure 9 shows CCFL system characteristics of the pressurizer surge line (PSL) models. The dimensionless liquid velocity $K_{\mathrm{L}}{ }^{*}$ of APEX (Takeuchi et al. [5]) (with $D=89 \mathrm{~mm}$ and steam-water) and the $1 / 10$-scale model by Futatsugi et al. [4] (with $D=30 \mathrm{~mm}$ and air-water), 


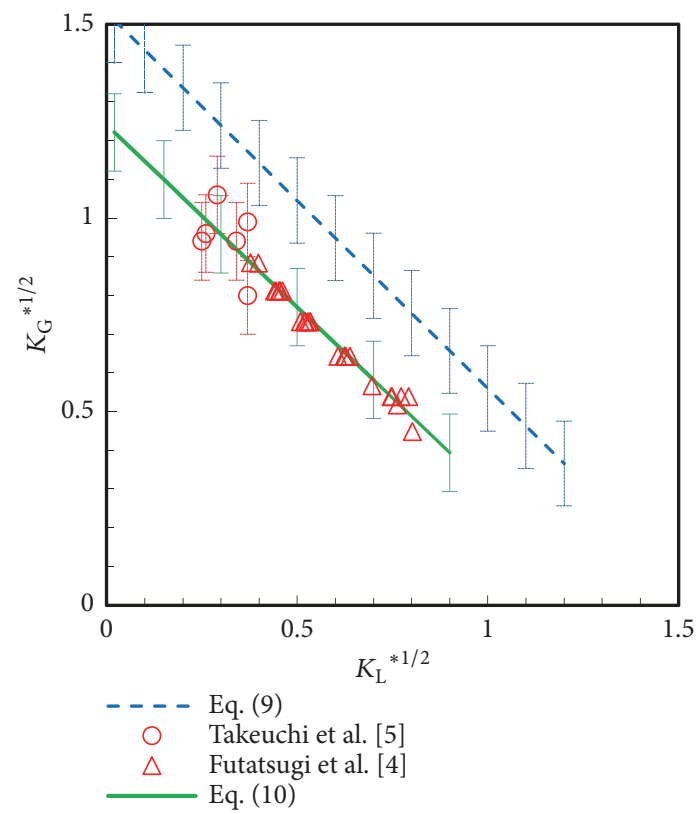

FIGURE 9: System characteristics of CCFL in a pressurizer surge line.

which simulated the pressurizer surge line models in the AP600 and a PWR, respectively, is smaller than that of (9) for the square top end in vertical pipes. From the integration test data in Figure 9, the following CCFL correlation was derived using the least-square method (Yamamoto et al. [8]):

$$
K_{\mathrm{G}}^{* 1 / 2}+0.94 K_{\mathrm{L}}^{* 1 / 2}=1.24 \pm 0.1 .
$$

Though the reason is not clear as to why the falling water velocity of the integration test data is smaller than that of (9), both sets of integration test data (Takeuchi et al. [5]; Futatsugi et al. [4]) can be well expressed by (10). Data used for deriving (10) are limited. Yu et al. [6,7] measured CCFL using the $1 / 4$-scale model $(D=90 \mathrm{~mm})$ of the pressurizer surge line model of the AP1000 with air-water and steamwater at atmospheric pressure. They changed the water level in the pressurizer simulator up to $h=900 \mathrm{~mm}$, and it affected CCFL. The effects of $h$ on $C_{\mathrm{K}}$ and $m$ in their experiments $[6,7]$ showed trends similar to those by Doi et al. [10] shown in Figure 4(b). Therefore, Figure 4(b), Figure 8(a), and Table 3 may be useful, when we evaluate data by Yu et al. $[6,7]$.

4.4. Technical Issues. Gas-liquid countercurrent flows in vertical pipes have been studied for over a half century, and it is well known that CCFL characteristics strongly depend on the shapes at the top and bottom ends (Wallis [15]; Bankoff and Lee [22]). We (Yamamoto et al. [23]) classified CCFL in vertical pipes depending on the limiting locations at the square bottom end (CCFL-L) and the square top end (CCFL-U) and inside the vertical pipe (CCFL-P), and we found that the characteristic lengths defined by (3) are $D, L$, and $D^{0.5} L^{0.5}$ for CCFL-L, CCFL-U, and CCFL-P, respectively. However, (1)-(4) only show effects of primary parameters, and actual CCFL characteristics are very complex and depend on some secondary parameters such as the diameters (cf.
Table 3), water levels, and fluid properties. Moreover, experimental data of CCFL differ among experimental facilities and conditions, and we have to consider some uncertainty among experimental data. Experiments at high pressure and temperature are few in number and effects of fluid properties as secondary parameters are still not clear. Wallis [15] showed that the liquid viscosity (which is not included in (1)-(4)) affects CCFL and he used the dimensionless viscosity number. We (Murase et al. [24]) used the viscosity ratio of the gas and liquid phases for CCFL-L, CCFL-U, and CCFL-P, but Ilyukhin et al. $[18,19]$ used the density ratio of the gas and liquid phases for CCFL with the protruding top and bottom ends. Therefore, CCFL correlations, which include effects of fluid properties, should be carefully used considering the application range. As the uncertainty of CCFL will increase on increasing the number of experimental data due to the inherent differences among the experimental facilities, the CCFL correlation should be derived using experimental data suitable for the system of concern.

Moreover, CCFL characteristics from integration tests expressed by (10) are different from those of (9) as shown in Figure 9. Therefore, engineering judgement is required to choose an appropriate CCFL correlation. At present, we recommend using the correlation equation (10), for the actual pressurizer surge line.

\section{Conclusions}

In this study, we evaluated effects of diameters on CCFL at the square top end in vertical pipes with and without the protruding end in the upper tank by using existing air-water data in the diameter range of $D=19-250 \mathrm{~mm}$ and with the water level in the upper tank up to $h=600 \mathrm{~mm}$. The results we obtained are as follows.

(1) We found that there was a strong relationship between the constant $C_{\mathrm{K}}$ and the slope $m$ in the Wallis-type CCFL correlation except for small diameters of $D=$ 19-25 mm with the water level at midlevel, where $C_{\mathrm{K}}$ increased with increasing $m$.

(2) On increasing the water level in the upper tank, $C_{\mathrm{K}}$ and $m$ in the Wallis-type CCFL correlation increased for $D=30 \mathrm{~mm}$. However, effects of the water level were complex and depended on the range of diameters and water levels.

(3) CCFL characteristics at the square top end were expressed by the Kutateladze parameters, and the constant and slope in the Wallis-type CCFL correlation were $C_{\mathrm{K}}=1.53 \pm 0.11$ and $m=0.97$, respectively, in the diameter range of $D=30-250 \mathrm{~mm}$. The $C_{\mathrm{K}}$ values were smaller than $C_{K}=1.53$ in the diameter range of $D=19-25 \mathrm{~mm}$.

The remaining technical issues for CCFL characteristics at the square top end are effects of fluid properties and application of the results to evaluate CCFL characteristics in the pressurizer surge line. 


\section{Nomenclature}

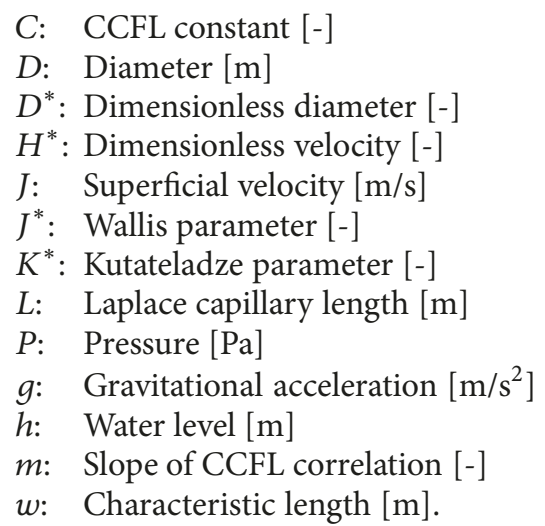

Greek Symbols

$$
\begin{aligned}
& \beta: \text { Exponent }[-] \\
& \rho: \text { Density }\left[\mathrm{kg} / \mathrm{m}^{3}\right] \\
& \sigma: \text { Surface tension }[\mathrm{N} / \mathrm{m}] \\
& \alpha: \text { Void fraction }[-] .
\end{aligned}
$$

\section{Subscripts}

G: Gas phase

$\mathrm{K}$ : Kutateladze parameter

L: Liquid phase

W: Wallis parameter.

\section{Data Availability}

The data used to support the findings of this study are included within the article.

\section{Conflicts of Interest}

The authors declare that they have no conflicts of interest.

\section{References}

[1] M. Murase, Y. Utanohara, T. Kusunoki, Y. Yamamoto, D. Lucas, and A. Tomiyama, "Prediction of countercurrent flow limitation and its uncertainty in horizontal and slightly inclined pipes," Nuclear Technology, vol. 197, no. 2, pp. 140-157, 2017.

[2] F. Mayinger, P. Weiss, and K. Wolfert, "Two-phase flow phenomena in full-scale reactor geometry," Nuclear Engineering and Design, vol. 145, no. 1-2, pp. 47-61, 1993.

[3] N. Minami, D. Nishiwaki, T. Nariai, A. Tomiyama, and M. Murase, "Countercurrent gas-liquid flow in a PWR hot leg under reflux cooling (I) air-water tests for 1/15-scale model of a PWR hot leg," Journal of Nuclear Science and Technology, vol. 47, no. 2, pp. 142-148, 2010.

[4] T. Futatsugi, C. Yanagi, M. Murase, S. Hosokawa, and A. Tomiyama, "Countercurrent Air-Water Flow in a Scale-Down Model of a Pressurizer Surge Line," Science and Technology of Nuclear Installations, vol. 2012, Article ID 174838, 7 pages, 2012.

[5] K. Takeuchi, M. Y. Young, and A. F. Gagnon, "Flooding in the pressurizer surge line of AP600 plant and analyses of APEX data," Nuclear Engineering and Design, vol. 192, no. 1, pp. 45-58, 1999.

[6] J. Yu, D. Zhang, L. Shi et al., "Experimental investigation of airwater CCFL in the pressurizer surge line of AP1000," Nuclear Technology, vol. 196, no. 3, pp. 614-640, 2016.

[7] J. Yu, D. Zhang, L. Shi et al., "Experimental research on the characteristics of steam-water counter-current flow in the Pressurizer Surge Line assembly," Experimental Thermal and Fluid Science, vol. 96, pp. 180-191, 2018.

[8] Y. Yamamoto, M. Murase, and A. Tomiyama, "Countercurrent flow limitation in a pressurizer surge line," Nuclear Engineering and Design, vol. 326, pp. 175-182, 2018.

[9] H. J. Richter, "Flooding in tubes and annuli," International Journal of Multiphase Flow, vol. 7, no. 6, pp. 647-658, 1981.

[10] T. Doi, T. Futatsugi, M. Murase, K. Hayashi, S. Hosokawa, and A. Tomiyama, "Countercurrent Flow Limitation at the Junction between the Surge Line and the Pressurizer of a PWR," Science and Technology of Nuclear Installations, vol. 2012, Article ID 754724, 9 pages, 2012.

[11] G. B. Wallis and J. T. Kuo, "The behavior of gas-liquid interfaces in vertical tubes," International Journal of Multiphase Flow, vol. 2 , no. 5-6, pp. 521-536, 1976.

[12] D. Bharathan, G. B. Wallis, and H. J. Richter, Air-water countercurrent annular flow, International Journal of Multiphase Flow, EPRI NP-1165, Electric Power Research Institute, Palo Alto, Calif., USA, 1979.

[13] K. Matsumura and F. Kaminaga, "Experimental Investigation of Rising Gas Bubble Characteristics from a Vertical Tube under CCFL Condition," Science and Technology of Nuclear Installations, vol. 2012, Article ID 785157, 15 pages, 2012.

[14] H. J. Richter, T. W. Lovell, and G. B. Wallis, The effects of scale on two-phase countercurrent flow flooding in vertical tubes, Final Report AT (49-24)-0329, Dartmouth College, 1977.

[15] G. B. Wallis, One-dimensional Two-phase Flow, McGraw Hill, New York, NY, USA, 1969.

[16] S. G. Bankoff, R. S. Tankin, M. C. Yuen, and C. L. Hsieh, "Countercurrent flow of air/water and steam/water through a horizontal perforated plate," International Journal of Heat and Mass Transfer, vol. 24, no. 8, pp. 1381-1395, 1981.

[17] G. B. Wallis and S. Makkenchery, "Hanging Film Phenomenon in Vertical Annular Two-Phase Flow," Journal of Fluids Engineering, vol. 96, no. 3, pp. 297-298, 1974.

[18] Yu. N. Ilyukhin, B. F. Balunov, E. L. Smirnov, and M. A. Gotovskii, "Hydrodynamic characteristics of two-phase annular flow in vertical channels (translated by authors)," Teplofiz. Vys. Temp, vol. 26, no. 5, pp. 923-931, 1988 (Russian).

[19] Y. N. Ilyukhin, S. V. Svetlov, S. B. Alekseev, V. O. Kukhtevich, and V. G. Sidorov, "The hydrodynamic characteristics of the process of "Flooding" under conditions of countercurrent flow of steam and water in vertical tubes," High Temperature, vol. 37, no. 3, pp. 463-469, 1999.

[20] D. Bharathan and G. B. Wallis, "Air-water countercurrent annular flow," International Journal of Multiphase Flow, vol. 9, no. 4, pp. 349-366, 1983.

[21] Y. Sudo, "Limitation of Falling Water in Countercurrent TwoPhase Flow in Vertical Circular Tubes," Transactions of the Japan Society of Mechanical Engineers Series B, vol. 60, no. 575, pp. 2566-2572, 1994.

[22] S. Bankoff and S. Lee, "Critical review of the flooding literature," NUREG/CR-3060, U.S. Nuclear Regulatory Commission, Washington DC, USA, 1983. 
[23] Y. Yamamoto, M. Murase, K. Hayashi, S. Hosokawa, and A. Tomiyama, "Counter-Current Flow Limitation inside Vertical Pipes," Japanese Journal of Multiphase Flow, vol. 30, no. 4, pp. 392-401, 2016.

[24] M. Murase, T. Kusunoki, Y. Yamamoto et al., "Effects of Fluid Properties on Countercurrent Flow Limitation in Vertical Pipes," Japanese Journal of Multiphase Flow, vol. 31, no. 2, pp. 152-161, 2017. 

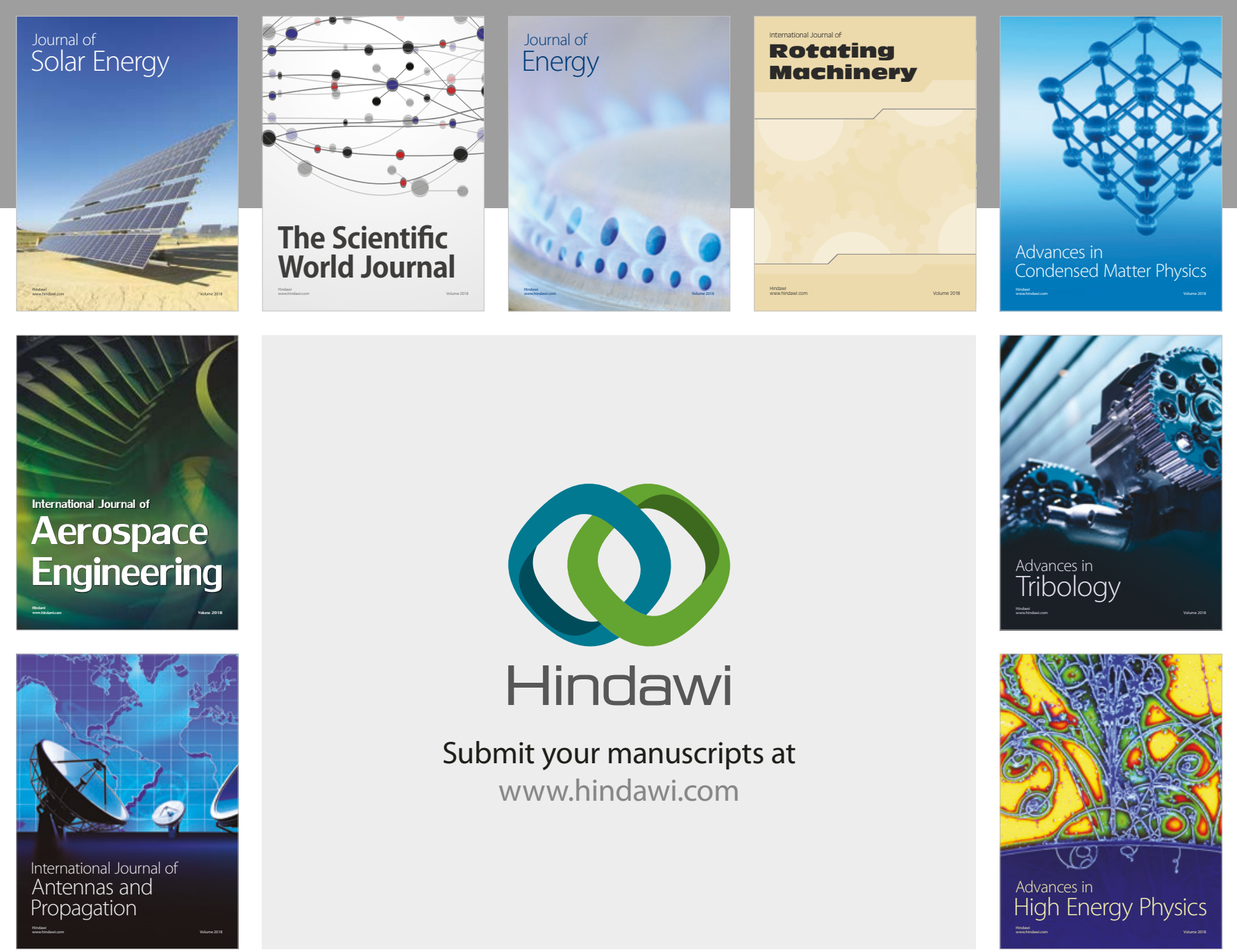

Submit your manuscripts at

www.hindawi.com
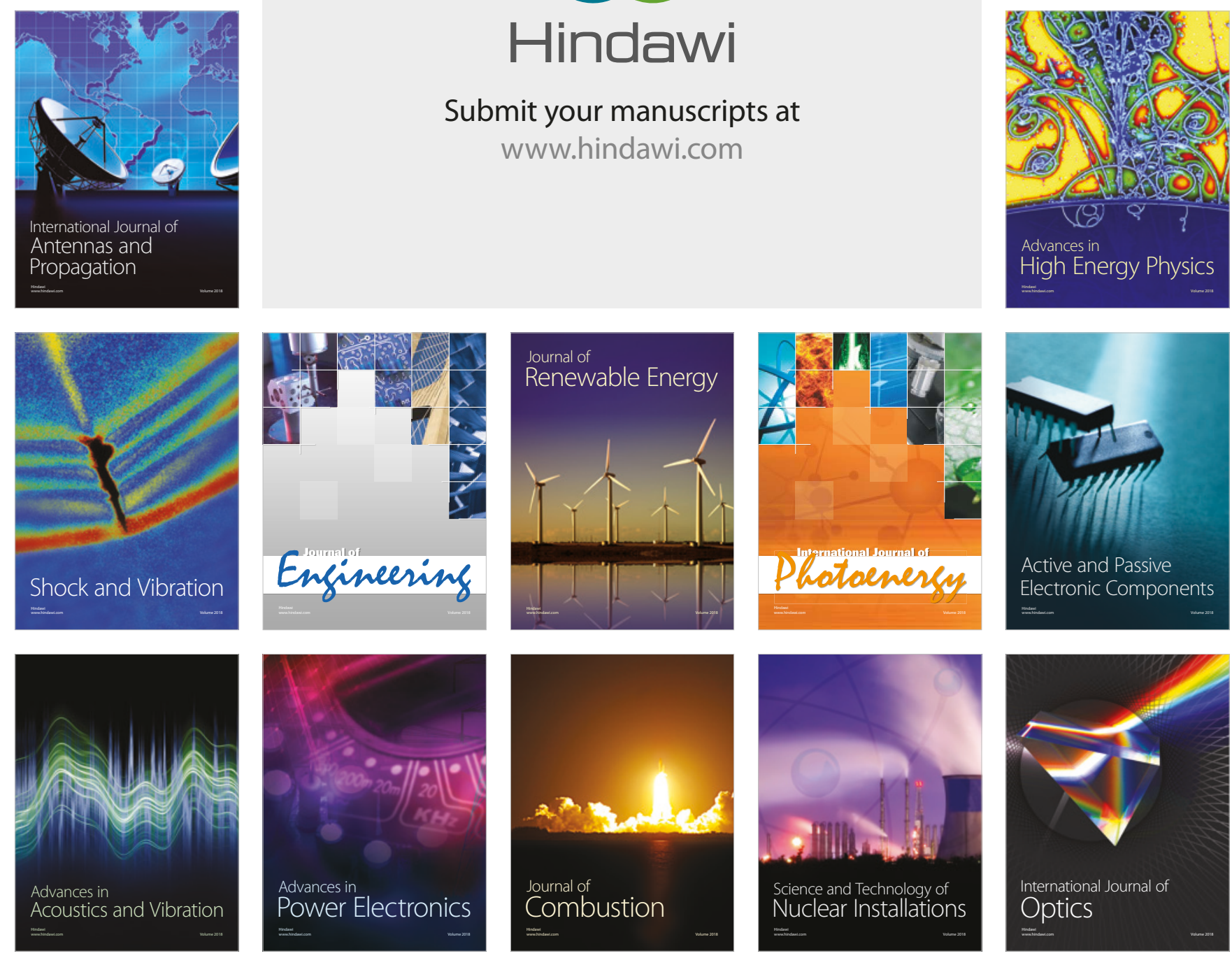